\title{
Ginsenoside Rb1 protects hippocampal neurons from high glucose-induced neurotoxicity by inhibiting GSK3 $\beta$-mediated CHOP induction
}

\author{
DI LIU ${ }^{1}$, HONG ZHANG ${ }^{2}$, WENJUAN GU ${ }^{1}$, YUQIN LIU ${ }^{2}$ and MENGREN ZHANG ${ }^{1}$ \\ ${ }^{1}$ Department of Traditional Chinese Medicine, Peking Union Medical College Hospital, Peking Union Medical College \\ and Chinese Academy of Medical Sciences, Beijing 100730; ${ }^{2}$ Department of Cell Resource Center, Institute of Basic \\ Medical Science, Peking Union Medical College and Chinese Academy of Medical Sciences, Beijing 100005, P.R. China
}

Received August 12, 2013; Accepted February 6, 2014

DOI: $10.3892 / \mathrm{mmr} .2014 .1958$

\begin{abstract}
Ginsenoside Rb1 is generally recognized as one of the principal bioactive ingredients in ginseng and shows neuroprotective effects in various neurons. Endoplasmic reticulum (ER) stress is considered to play an important role in numerous neurodegenerative disorders. Recently, glucogen synthase kinase $3 \beta$ (GSK3 $\beta$ ) was reported to regulate ER stress-induced C/EBP homologous protein (CHOP) in neuronal cells. Therefore, in this study, we investigated the effects of ginsenoside Rb1 on GSK3 $\beta$ mediated ER stress in high glucose-treated hippocampal neurons. Results from the MTT assay showed that treatment with $1 \mu \mathrm{M} \mathrm{Rb} 1$ for $72 \mathrm{~h}$ protected neurons from high glucose-induced cell injury. Using western blot analysis, we found that treatment with Rb1 effectively inhibited the phosphorylation of the high glucose-induced protein kinase RNA-like ER kinase (PERK) and of GSK $3 \beta$, and reduced the level of the CHOP protein. The levels of these proteins were also decreased by treatment with the GSK3 $\beta$ inhibitor Licl. Rb1 also significantly decreased the mRNA expression of the gene $C H O P$, as shown by quantitative RT-PCR analysis. Taken together, the present results suggested that $\mathrm{Rb} 1$ may protect neurons from high glucose-induced cell damage by inhibiting GSK3 $\beta$-mediated CHOP induction, providing a potentially new strategy for preventing and treating cognitive impairment caused by diabetes.
\end{abstract}

Correspondence to: Professor Mengren Zhang, Department of Traditional Chinese Medicine, Peking Union Medical College Hospital, Peking Union Medical College and Chinese Academy of Medical Sciences, No. 1 Shuaifuyuan, Dongcheng, Beijing 100730, P.R. China

E-mail: xh02742@163.com

Key words: ginsenoside Rb1, high glucose, GSK3 $\beta$, endoplasmic reticulum stress, hippocampal neurons

\section{Introduction}

Diabetes is a thriving disease throughout the world and evidence from several epidemiological studies suggests that it is an independent risk factor for cognitive dysfunction (1). Patients with type 1 and type 2 diabetes show performance decrements on measures of executive functioning (2). Substantial evidence supports that diabetic cognitive impairment is associated with dysfunctions of the hippocampus, the major area associated with learning and memory (3-5). Experimental studies in animal models and in humans have shown that hyperglycemia is the main contributor of diabetic cognitive impairment, which can alter hippocampal synaptic plasticity and cause decreased neuronal densities in the CA-1 region of the hippocampus and degeneration and apoptosis of hippocampal neurons $(4,6,7)$.

Endoplasmic reticulum (ER) stress, often resulting from the cellular accumulation of misfolded proteins, triggers a cellular stress response known as the unfolded protein response (UPR), which is intended to protect the cell (8). UPR is characterized by translational attenuation, synthesis of ER chaperone proteins and transcriptional induction of genes including those encoding the three ER transmembrane receptors, i.e., protein kinase RNA (PKR)-like ER kinase (PERK), inositol requiring enzyme-1 (IRE1) and activating transcription factor (ATF6) (8). If homeostasis cannot be reestablished or ER function is severely impaired, apoptosis signaling pathways are triggered by ER stress, including the C/EBP homology protein (CHOP), caspase-12 and c-Jun $\mathrm{N}$-terminal kinase pathways $(9,10)$. The CHOP protein, shown to play a critical role in ER stress-induced apoptosis, can be induced by PERK translation, and has been involved in promoting neuronal death in numerous neurodegenerative diseases (11). Recently, CHOP-dependent ER stress-mediated apoptosis was reported to be involved in hyperglycemia-induced impairment of hippocampal synapses and neurons (12). Regulation of the ER stress-induced execution phase of apoptosis is not completely understood, but clearly involves glycogen synthase kinase-3 $\beta$ (GSK3 $\beta$ ), which regulates ER stress-induced CHOP expression in neuronal cells (13).

Ginseng (botanical name: Panax ginseng C.A. Meyer) is one of the most common herbal remedies and has long been 
used in traditional Chinese medicine to restore and enhance benefits for patients. Ginsenoside Rb1 is generally recognized as one of the principal bioactive ingredients in ginseng and has been shown to possess neuroprotective properties in numerous studies. Ginsenoside Rb1 can increase the proliferation of Schwann cells and the expression and secretion of nerve growth factor (NGF) and brain-derived neurotrophic factor (BDNF) from these cells (14), prevent $\mathrm{MPP}^{+}$-induced apoptosis in $\mathrm{PC} 12$ cells (15) and upregulate cell genesis in hippocampal subregions, thus enhancing spatial learning and memory in rats (16). However, whether ginsenoside Rb1 has neuroprotective effects on high glucose-treated hippocampal neurons, and the mechanism underlying these effects remain unclear. Therefore, in the present study, we investigated the effects of ginsenoside Rb1 on high glucose-induced cytotoxicity in primary cultured rat hippocampal neurons by detecting cell viability, and further studied the underlying mechanisms by examining phosphorylated (p)-PERK, PERK, p-GSK3 $\beta$ (Tyr216), GSK3 $\beta$ and CHOP protein expression, as well as $C H O P$ mRNA expression.

\section{Materials and methods}

Reagents. Ginsenoside Rb1 standard was purchased from the National Institute for the Control of Pharmaceutical and Biological Produces (Beijing, China). The chemical structure of ginsenoside Rb1 (2-O- $\beta$-glucopyranosyl- $(3 \beta, 12 \beta)-20-[(6-\mathrm{O}-\beta$ -D-glucopyranosyl- $\beta$-D-glucopyranosyl) oxy]-12-hydroxydammar-24-en-3-yl $\beta$-D-glucopyranoside) is shown in Fig. 1. All cell culture reagents were from the Peking Union Cell Resource Center (Beijing, China). Sprague-Dawley (SD) rats, $<24$ h old, were purchased from the Peking University Health Science Center (Beijing, China). Anti-p-PERK, -PERK, -p-GSK3 $\beta$, -GSK3 $\beta$ and -CHOP antibodies were purchased from Santa Cruz Biotechnology, Inc. (Santa Cruz, CA, USA).

Cell culture and treatments. All experimental procedures were performed in accordance with the experimental standards of the Chinese Academy of Medical Sciences, as well as with international guidelines on the ethical treatment of animals. Primary hippocampal neuronal cultures were prepared as previously described $(17,18)$ with some modifications. The Animal Welfare Committee of Peking Union Medical College Hospital and Chinese Academy of Medical Sciences (Beijing, China) approved the animal protocols. Hippocampi were dissected from the brain on ice and minced in sterile ice-cold D-Hanks solution, with the blood vessels and meninges carefully removed. The tissues were digested with $0.25 \%$ trypsin for $15 \mathrm{~min}$ at $37^{\circ} \mathrm{C}$, and the digestion reaction was terminated by adding $5 \mathrm{ml}$ fetal bovine serum (FBS). The cell suspension was passed through a 200 mesh cell strainer and separated by density gradient centrifugation at $702.4 \mathrm{x}$ g for $20 \mathrm{~min}$. Then, the cell suspension, containing the desired cell fractions in D-Hanks' solution, was centrifuged for $5 \mathrm{~min}$ at $395.1 \mathrm{x} \mathrm{g}$ and resuspended in $15-20 \mathrm{ml}$ Dulbecco's modified Eagle's medium (DMEM). The cells were then plated on poly-D-lysine (Sigma-Aldrich, St. Louis, MO, USA)-coated glass coverslips, 96-well plates or 24-well plates at a density of $5 \times 10^{5}$ cells $/ \mathrm{ml}$. The cell density was previously determined using a hemacytometer. The cells were maintained at $37^{\circ} \mathrm{C}$ in a humidified $5 \% \mathrm{CO}_{2}$ incubator (Thermo Scientific Forma, Waltham, MA, USA). Neurons were cultured in DMEM

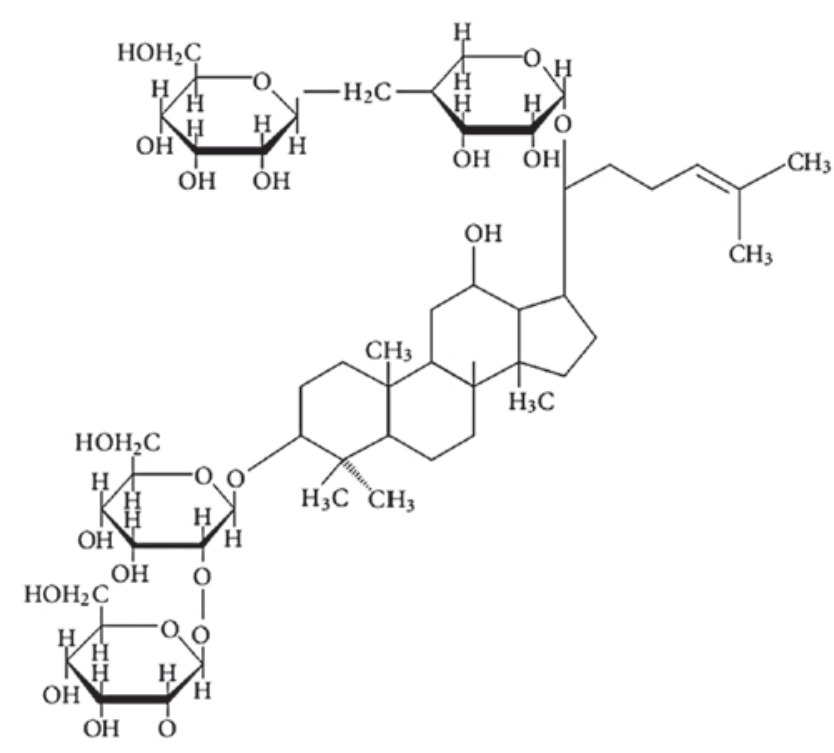

Figure 1. Chemical structure of ginsenoside Rb1.

medium and supplemented with 10\% FBS, $20 \mathrm{mmol} / 1$ sodium pyruvate, $1 \mathrm{mmol} / \mathrm{l}$ glutamine, $100 \mathrm{U} / \mathrm{ml}$ penicillin, $100 \mu \mathrm{g} / \mathrm{ml}$ streptomycin and $10 \mathrm{ng} / \mathrm{ml}$ NGF (Millipore, Billerica, MA, USA). Ginsenoside Rb1 standard (1 $\mu \mathrm{M})$, high glucose (final concentration, $50 \mathrm{mM})$ and Licl (1 mM; Sigma-Aldrich) were added to the neurons for $72 \mathrm{~h}$.

Cell viability assay. After exposure to $50 \mathrm{mM}$ glucose and ginsenoside or $\mathrm{LiCl}$ for $72 \mathrm{~h}$, cell viability was determined using the MTT assay system (Peking Union Cell Resource Center). Briefly, the cells were plated on 96-well culture plates at a density of $5 \times 10^{5}$ cells $/ \mathrm{ml}$. Then, MTT reagent $(100 \mu \mathrm{l}$ of a $5 \mathrm{mg} / \mathrm{ml}$ solution) was added to each well (1:10) and incubated for $4 \mathrm{~h}$ at $37^{\circ} \mathrm{C}$. Following incubation, the medium was removed and the precipitated dye was dissolved in $100 \mu \mathrm{l}$ of dimethylsulfoxide solution for another $30 \mathrm{~min}$ at $37^{\circ} \mathrm{C}$. The OD value of each sample was measured at $570 \mathrm{~nm}$ using a Multiskan 3 ELISA Reader (Thermo Fisher Scientific, Inc., Waltham, MA, USA). Cell viability (\%) was calculated as follows: $\mathrm{OD}_{\text {treated group }} / \mathrm{OD}_{24 \text { h control group }} \mathrm{X} 100$.

Western blot analysis. Following treatment as described above, total protein was extracted from cells lysed with RIPA buffer (50 mM Tris- $\mathrm{HCl}$ at $\mathrm{pH} 7.4,150 \mathrm{mM} \mathrm{NaCl}, 1 \%$ sodium deoxycholate, $1 \mathrm{mM}$ EDTA, $0.1 \% \mathrm{SDS}, 5 \mu \mathrm{g} / \mathrm{ml}$ aprotinin and $5 \mu \mathrm{g} / \mathrm{ml}$ leupeptin). The protein concentration of the supernatants was determined with the DC protein assay kit 1 (Bio-Rad, Hercules, CA, USA) and then adjusted to the same concentration with $0.9 \% \mathrm{NaCl}$. A total of $40 \mu \mathrm{g}$ of protein was resolved by $15 \%$ SDS-polyacrylamide gel electrophoresis (PAGE) and then transferred to a nitrocellulose membrane. The membrane was blocked with $5 \%$ skim milk in Tris-buffered saline with $0.1 \%$ Tween-20 (TBST) for $1 \mathrm{~h}$ at room temperature before overnight incubation with primary antibodies specific to p-PERK (1:100), PERK (1:100), p-GSK3 $\beta$ (1:200), GSK3 $\beta$ (1:200) and CHOP (1:200) in 5\% skim milk-TBST. The membrane was then rinsed three times in TBST and incubated with horseradish peroxidase-conjugated secondary $\operatorname{IgG}(1: 1,000)$ in TBST for 

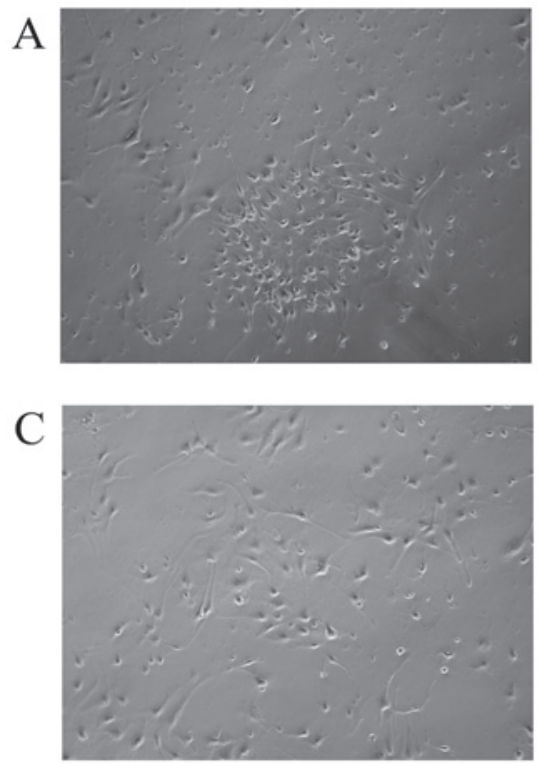

B

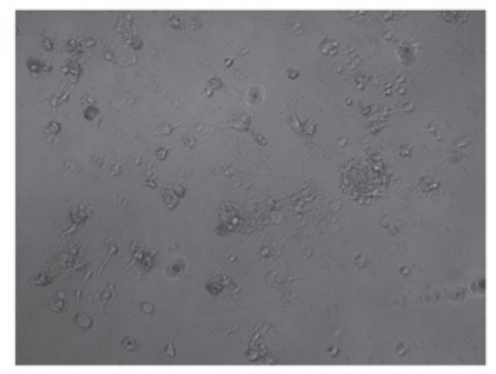

$\mathrm{D}$

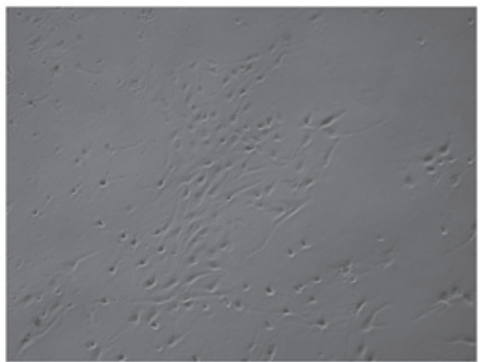

Figure 2. Neuronal morphological changes in the (A) control, (B) high glucose, (C) ginsenoside Rb1+ high glucose and (D) Licl+ high glucose groups.

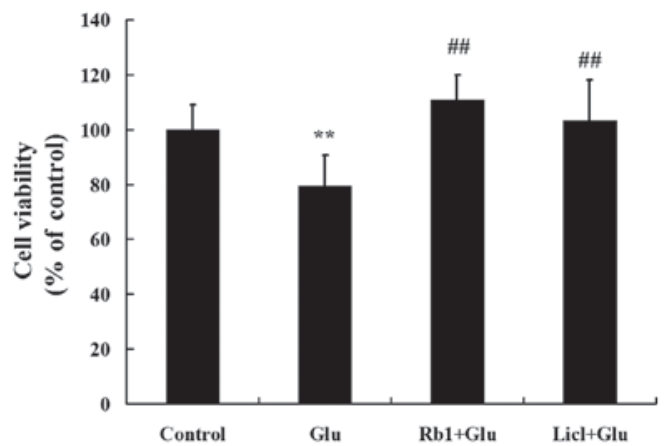

Figure 3. Effects of ginsenoside Rb1 on viability of high glucose-treated hippocampal neurons. Hippocampal neurons were treated with ginsenoside $\mathrm{Rb} 1(1 \mu \mathrm{M})$ or Licl $(1 \mathrm{mM})$ in the presence of $50 \mathrm{mM}$ glucose for $72 \mathrm{~h}$. Hippocampal neurons in the control group were treated with normal medium which contained $25 \mathrm{mM}$ glucose for $72 \mathrm{~h}$. Cell viability was measured using the MTT assay. Results are presented as the mean \pm SD of at least three independent experiments, and are expressed as a percentage of the control; ${ }^{* *} \mathrm{P}<0.01$, as compared to the control group; ${ }^{\# \#} \mathrm{P}<0.01$, as compared to the high glucose group.

$1.5 \mathrm{~h}$, followed by a final series of rinses in TBST. Protein bands were visualized using an enhanced chemiluminescence (ECL) kit (Roche Diagnostics GmbH, Penzberg, Germany) and were quantified using the Quantity One software (Bio-Rad).

Real-time PCR analysis. Total RNA was extracted from cells using the TRIzol reagent according to the manufacturer's instructions. The concentration and purity of RNA were determined spectrophotometrically at 260 and $280 \mathrm{~nm}$. Single-strand cDNA was prepared from $2 \mu \mathrm{g}$ of total RNA according to the protocol of the kit. The following primers were designed using Primer Premier 5.0 software (http://www.premierbiosoft.com/): CHOP, 5'- CCTAGC T T G GCT GACT GAGG-3', 5'-CTGCTCCTTCTCCTTCATGC-3'; GAPDH, 5'-TGG TATCGTGGAAGGACTCA-3', 5'-CCAGATGAGGCAGGGA TGAT-3'. Briefly, the PCR reaction was carried out in a $20 \mu \mathrm{l}$ final volume containing: $10 \mu 1$ of $2 \mathrm{X} \mathrm{SYBR}^{\circledR}$-Green I master mix, $1 \mu \mathrm{l}$ of each primer $(10 \mu \mathrm{M}), 2 \mu \mathrm{l}$ of $1 \mu \mathrm{g} / \mu \mathrm{l}$ cDNA template, and $6 \mu \mathrm{l}$ of $0.1 \%$ diethylpyrocarbonate-treated water. The PCR conditions were as follows: initial denaturation at $95^{\circ} \mathrm{C}$ for $120 \mathrm{sec}$, followed by 40 cycles of denaturation at $95^{\circ} \mathrm{C}$ for $20 \mathrm{sec}$, annealing at $60^{\circ} \mathrm{C}$ for $20 \mathrm{sec}$, and extension at $72^{\circ} \mathrm{C}$ for $30 \mathrm{sec}$. A melting curve was obtained after amplification by holding the temperature at $65^{\circ} \mathrm{C}$ for $20 \mathrm{sec}$, followed by a gradual increase in temperature to $95^{\circ} \mathrm{C}$ at a rate of $0.5^{\circ} \mathrm{C} / \mathrm{sec}$. The $G A P D H$ mRNA level was used as an internal quantitative control, allowing to normalize the level of each target gene transcript.

Statistical analysis. Data are expressed as mean \pm SD. Differences between groups were examined for statistical significance using a one-way ANOVA and Dunnett's tests. $\mathrm{P}<0.05$ was considered to indicate significant differences.

\section{Results}

Neuronal morphological changes. Neuronal morphological changes were assessed under an inverted microcope. As shown in Fig. 2, hippocampal neurons treated with normal medium (control) exhibited large, vacuole-free cell bodies, with elaborate networks of neurites. However, exposure to $50 \mathrm{mM}$ glucose for $72 \mathrm{~h}$ resulted in obvious cell loss, with the disappearance of neurites, appearance of disrupted membranes and shrinkage of cell bodies. By contrast, cultures exposed to the same concentration of glucose in the presence of ginsenoside $\mathrm{Rb} 1(1 \mu \mathrm{M})$ appeared markedly preserved, indicating that $\mathrm{Rb} 1$ has significant cytoprotective effects against high glucose insult. Licl $(1 \mathrm{mM})$, which is a GSK3 $\beta$ inhibitor, also showed cytoprotective effects against high glucose treatment.

Neuroprotective effects of ginsenoside Rbl on cytotoxicity induced by high glucose. To verify the protective effects of ginsenoside $\mathrm{Rb} 1$ on hippocampal neurons exposed to a high level of glucose, cell viability was evaluated by determining the percentage of MTT reduction. As shown in Fig. 3, $50 \mathrm{mM}$ of glucose induced a significant decrease in cell viability 
A

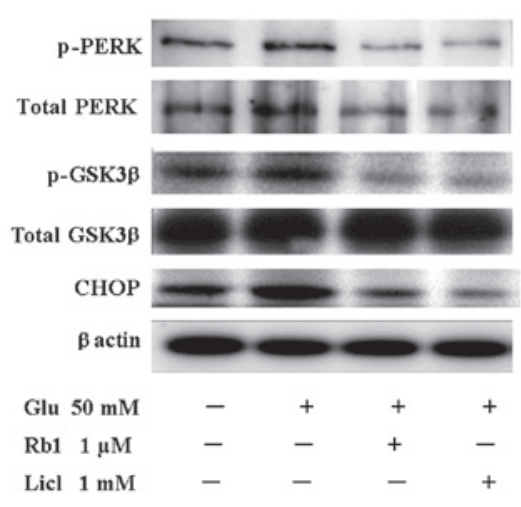

B

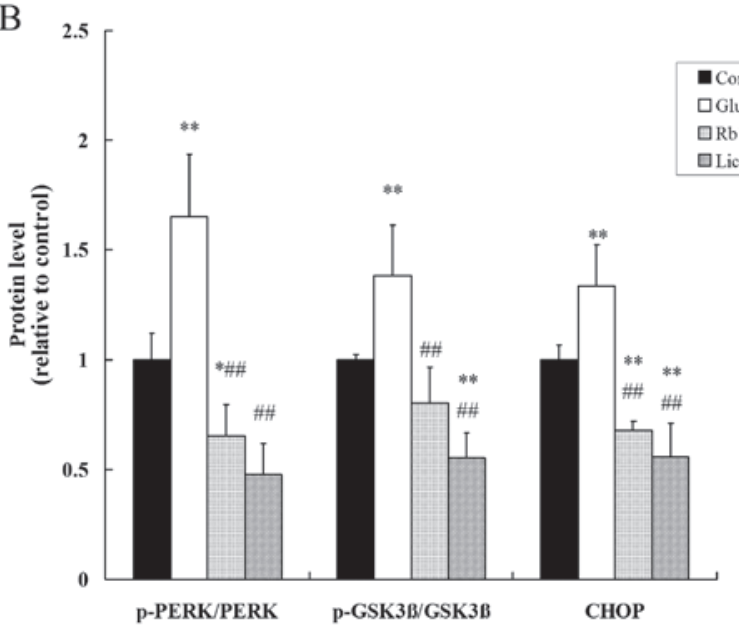

$\mathrm{C}$

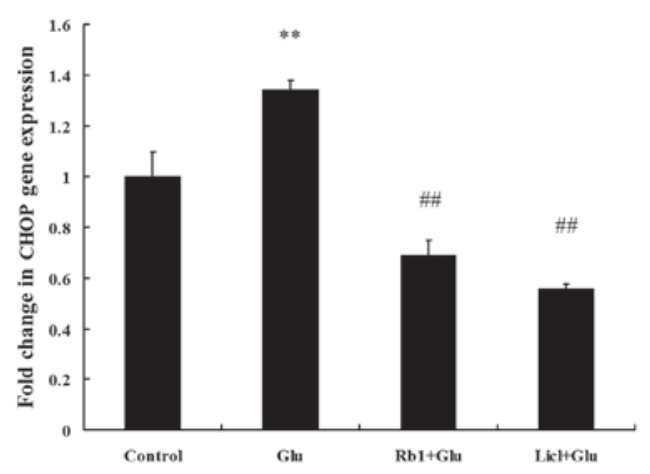

Figure 4. Effects of ginsenoside Rb1 on glucogen synthase kinase $3 \beta$ (GSK3 $\beta$ )-mediated ER stress in high glucose-treated hippocampal neurons. (A) Representative western blot showing the effects of ginsenoside Rb1 during high glucose exposure on activation (phosphorylation, $\mathrm{p}$ ) of PERK, GSK3 $\beta$ and CHOP. (B) Semi-quantitative analysis of protein levels. $\beta$-actin was used as the loading control. (C) Histogram of quantitative RT-PCR results indicating the fold changes in the $C H O P$ mRNA level, normalized to $G A P D H$, relative to the control. Hippocampal neurons in the control group were treated with normal medium which contained $25 \mathrm{mM}$ glucose for $72 \mathrm{~h}$. Results are presented as mean $\pm \mathrm{SD}$ of three independent experiments. ${ }^{*} \mathrm{P}<0.05$, as compared to the control group; ${ }^{* *} \mathrm{P}<0.01$, as compared to the control group; ${ }^{\# \#} \mathrm{P}<0.01$, as compared to the high glucose group.

compared to the control group. However, treatment with $1 \mu \mathrm{M}$ of ginsenoside Rb1 significantly improved cell viability in high glucose-treated hippocampal neurons.

Effects of ginsenoside Rb1 on GSK3 $\beta$-mediated ER stress in high glucose-treated hippocampal neurons. To examine the activation of ER stress in high glucose-induced cell damage conditions, the phosphorylation level of PERK and GSK3 $\beta$ and the expression of $\mathrm{CHOP}$ were assessed by western blot analysis (Fig. 4A). Compared to the control, cells exposed to a high level of glucose for $72 \mathrm{~h}$ showed significantly increased $p$-PERK/PERK and $p-G S K 3 \beta / G S K 3 \beta$ ratios and an increased level of the CHOP protein (Fig. 4B). By contrast, treatment with ginsenoside Rb1 effectively attenuated the high glucose-induced phosphorylation of PERK and GSK $3 \beta$ without affecting the levels of the unphosphorylated forms of the enzymes, and reduced the level of CHOP (Fig. 4A and B).

We then investigated the effect of ginsenoside $\mathrm{Rb} 1$ on CHOP gene expression using quantitative RT-PCR analysis. As shown in Fig. $4 \mathrm{C}$, exposure to high glucose for $72 \mathrm{~h}$ resulted in a significant increase in $\mathrm{CHOP}$ gene expression compared to the control. However, when cells were treated with ginsenoside $\mathrm{Rb} 1$, the $C H O P$ mRNA level was significantly decreased compared to the high glucose group.

To investigate the hypothesis that active GSK $3 \beta$ is required for the increase in CHOP following ER stress, hippocampal neurons were treated with the inhibitor of GSK3 $\beta$ (Licl) in the presence of a high level of glucose for $72 \mathrm{~h}$. Western blot analysis and RT-PCR measurements confirmed that Licl inhibits high glucose-induced phosphorylation of PERK and GSK3 $\beta$, as well as CHOP protein and mRNA expression (Fig. 4). The MTT assay indicated that cell viability is also significantly improved by treatment with Licl in high glucose-treated hippocampal neurons (Fig. 3).

\section{Discussion}

Ginseng has served as an important component of numerous traditional Chinese medicine prescriptions for thousands of years and is now popular as a natural medicine worldwide. Ginsenoside Rb1 is the major pharmacologically active ingredient of ginseng, and its anti-aging and neuroprotective effects have been often reported. Cell-based studies showed that ginsenoside $\mathrm{Rb} 1$ protects hippocampal neurons from glutamate-induced neurodegeneration (19) and promotes cell survival, enhanced neurite outgrowth and synaptic marker expression during differentiation of human neural stem cells (20). However, the precise biological functions and underlying mechanisms of ginsenoside $\mathrm{Rb}$ are still largely unknown.

The present study evaluated ginsenoside $\mathrm{Rb} 1$ as a protective agent against high glucose-induced neurotoxicity. Ginsenoside $\mathrm{Rb} 1$ attenuated the neuronal loss and improved cell viability in high glucose-treated hippocampal neurons. We further provide evidence that this neuroprotective effect may involve the inhibition of GSK3 $\beta$-mediated ER stress induced by high glucose.

Accumulating evidence suggests that diabetes is associated with cognitive deficits, and previous studies have shown that hyperglycemia has toxic effects and can lead to functional and structural abnormalities in the brain, especially the hippocampus $(2,21)$. Hippocampal atrophy is evident early in the course of the disease, and has even been documented in elderly individuals with prediabetes (22). In animal models, diabetes is associated with changes in the hippocampal synaptic plasticity, molecular changes in hippocampal neurons, including pre- and post-synaptic structures, and degeneration of hippocampal neurons (23-25). Therefore, we used hippocampal neurons exposed to high glucose as an in vitro model to 
investigate the neuroprotective effects of ginsenoside $\mathrm{Rb} 1$ on high glucose-induced neurotoxicity, as well as the underlying mechanism. Consistent with a previous study (26), the results from morphological observations and the cell viability assay showed that a high level of glucose induces cell damage. Treatment of hippocampal neurons with ginsenoside Rb1 significantly attenuated the high glucose-induced morphological changes in the neurons and the decrease in cell viability, suggesting that ginsenoside Rb1 can revert the neurotoxicity induced by high glucose.

Hyperglycemia plays a critical role in hippocampus dysfunction, however, the precise cellular mechanisms underlying this effect have not yet been fully elucidated. A recent study on a murine model of type 2 diabetes suggested that ER stress is activated in the hippocampus (27), while another study indicated that CHOP-dependent ER stress-mediated apoptosis may be involved in high glucose-induced neuronal damage (12). In rats it has also been reported that diabetes exacerbates ischemic brain injury by increasing ER stress and apoptosis, associated with enhanced CHOP induction (28). CHOP is a pro-apoptotic protein that plays a major role in ER stress-induced apoptosis. In neuronal cells, GSK3 $\beta$ was shown to regulate ER stress-induced CHOP expression, and inhibition of GSK $3 \beta$ effectively reduced the expression of CHOP (13). Therefore, we hypothesized that GSK3 $\beta$-mediated CHOP induction may be involved in high glucose-induced cell injury. In this study, we observed a significantly higher expression of p-PERK, p-GSK $3 \beta$ and CHOP in high glucose-treated hippocampal neurons, which was effectively decreased by the GSK3 $\beta$ inhibitor, indicating that GSK3 $\beta$-mediated CHOP signaling is involved in mediating the effects of high glucose on cell morphology and structure. The results of this study showed that high glucose-induced activation of CHOP is significantly inhibited by treatment with the ginsenoside Rb1 and by the GSK3 $\beta$ inhibitor, which suggests that ginsenoside $\mathrm{Rbl}$ can protect from high glucose-induced neurotoxicity via inhibition of the GSK3 $\beta$ pathway.

In summary, our study demonstrated that ginsenoside Rb1 protects hippocampal neurons from high glucose-induced cytotoxicity. The mechanisms underlying this neuroprotective effect may involve, at least in part, the inhibition of GSK3 $\beta$-mediated CHOP induction. To better understand diabetic cognitive impairment, the contribution of proteins downstream of CHOP in high glucose-induced neurotoxicity merit further investigation. Additional investigations on ginsenoside $\mathrm{Rb} 1$ is expected to further clarify the mechanisms by which this substance protects the neurons from damage, which may contribute to the development of potential therapeutic applications for this potent neuroprotective agent.

\section{Acknowledgements}

We are grateful to Professor Hongchao Yin and Bei Gu for their helpful technical assistance and scientific suggestions.

\section{References}

1. Cukierman T, Gerstein HC and Williamson JD: Cognitive decline and dementia in diabetes - systematic overview of prospective observational studies. Diabetologia 48: 2460-2469, 2005.

2. McCrimmon RJ, Ryan CM and Frier BM: Diabetes and cognitive dysfunction. Lancet 379: 2291-2299, 2012.
3. Revsin Y, Saravia F, Roig P, et al: Neuronal and astroglial alterations in the hippocampus of a mouse model for type 1 diabetes. Brain Res 1038: 22-31, 2005.

4. Li ZG, Zhang W, Grunberger G and Sima AA: Hippocampal neuronal apoptosis in type 1 diabetes. Brain Res 946: 221-231, 2002.

5. Gold SM, Dziobek I and Sweat V, et al: Hippocampal damage and memory impairments as possible early brain complications of type 2 diabetes. Diabetologia 50: 711-719, 2007.

6. Kamal A, Biessels GJ, Urban IJ and Gispen WH: Hippocampal synaptic plasticity in streptozotocin-diabetic rats: impairment of long-term potentiation and facilitation of long-term depression. Neuroscience 90: 737-745, 1999.

7. Stranahan AM, Arumugam TV, Cutler RG, Lee K, Egan JM and Mattson MP: Diabetes impairs hippocampal function through glucocorticoid-mediated effects on new and mature neurons. Nat Neurosci 11: 309-317, 2008.

8. Ron D and Walter P: Signal integration in the endoplasmic reticulum unfolded protein response. Nat Rev Mol Cell Bio 8: 519-529, 2007.

9. Tabas I and Ron D: Integrating the mechanisms of apoptosis induced by endoplasmic reticulum stress. Nat Cell Biol 13: 184-190, 2011.

10. Liu D, Zhang M and Yin H: Signaling pathways involved in endoplasmic reticulum stress-induced neuronal apoptosis. Int J Neurosci 123: 155-162, 2013.

11. Yang $\mathrm{W}$ and Paschen $\mathrm{W}$ : The endoplasmic reticulum and neurological diseases. Exp Neurol 219: 376-381, 2009.

12. Zhang $\mathrm{X}, \mathrm{Xu} \mathrm{L}, \mathrm{He} \mathrm{D}$ and Ling S: Endoplasmic reticulum stress-mediated hippocampal neuron apoptosis involved in diabetic cognitive impairment. Biomed Res Int 2013: 924327, 2013.

13. Meares GP, Mines MA, Beurel E, et al: Glycogen synthase kinase-3 regulates endoplasmic reticulum (ER) stress-induced CHOP expression in neuronal cells. Exp Cell Res 317: 1621-1628, 2011.

14. Liang W, Ge S, Yang L, et al: Ginsenosides Rb1 and Rg1 promote proliferation and expression of neurotrophic factors in primary Schwann cell cultures. Brain Res 1357: 19-25, 2010.

15. Hashimoto R, Yu J, Koizumi H, Ouchi Y and Okabe T: Ginsenoside Rb1 prevents $\mathrm{MPP}^{+}$-induced apoptosis in $\mathrm{PC} 12$ cells by stimulating estrogen receptors with consequent activation of ERK1/2, Akt and inhibition of SAPK/JNK, p38 MAPK. Evid Based Complement Alternat Med 2012: 693717, 2012.

16. Liu L, Hoang-Gia T, Wu H, et al: Ginsenoside Rb1 improves spatial learning and memory by regulation of cell genesis in the hippocampal subregions of rats. Brain Res 1382: 147-154, 2011.

17. Kaech S and Banker G: Culturing hippocampal neurons. Nat Protoc 1: 2406-2415, 2007.

18. Brewer GJ and Torricelli JR: Isolation and culture of adult neurons and neurospheres. Nat Protoc 2: 1490-1498, 2007.

19. Radad K, Gille G, Moldzio R, Saito H and Rausch WD: Ginsenosides Rb1 and Rg1 effects on mesencephalic dopaminergic cells stressed with glutamate. Brain Res 1021: 41-53, 2004.

20. Wang L and Kisaalita WS: Administration of BDNF/ginsenosides combination enhanced synaptic development in human neural stem cells. J Neurosci Methods 194: 274-282, 2011.

21. Gispen WH and Biessels GJ: Cognition and synaptic plasticity in diabetes mellitus. Trends Neurosci 23: 542-549, 2000.

22. Convit A, Wolf OT, Tarshish C and de Leon MJ: Reduced glucose tolerance is associated with poor memory performance and hippocampal atrophy among normal elderly. Proc Natl Acad Sci USA 100: 2019-2022, 2003.

23. Alvarez EO, Beauquis J and Revsin Y, et al: Cognitive dysfunction and hippocampal changes in experimental type 1 diabetes. Behav Brain Res 198: 224-230, 2009.

24. Guo J, Yu C and Li H, et al: Impaired neural stem/progenitor cell proliferation in streptozotocin-induced and spontaneous diabetic mice. Neurosci Res 68: 329-336, 2010

25. Artola A, Kamal A, Ramakers GM, Biessels GJ and Gispen WH: Diabetes mellitus concomitantly facilitates the induction of long-term depression and inhibits that of long-term potentiation in hippocampus. Eur J Neurosci 22: 169-178, 2005.

26. Gaspar JM, Castilho A, Baptista FI, Liberal J and Ambrosio AF: Long-term exposure to high glucose induces changes in the content and distribution of some exocytotic proteins in cultured hippocampal neurons. Neuroscience 171: 981-992, 2010.

27. Sims-Robinson C, Zhao S, Hur J and Feldman EL: Central nervous system endoplasmic reticulum stress in a murine model of type 2 diabetes. Diabetologia 55: 2276-2284, 2012.

28. Srinivasan K and Sharma SS: Augmentation of endoplasmic reticulum stress in cerebral ischemia/reperfusion injury associated with comorbid type 2 diabetes. Neurol Res 33: 858-865, 2011. 